\title{
Simplification of Flavour Combinatorics in the Evaluation of Hadronic Processes
}

\author{
E.E. Boos*, V.A. Ilyin* and A.N. Skachkova*† \\ *Institute of Nuclear Physics, Moscow State University, 119899 Moscow, Russia \\ ${ }^{\dagger} J o i n t$ Institute for Nuclear Research, 141980 Dubna, Russia
}

\begin{abstract}
A serious computational problem in the evaluation of hadronic collision processes is connected with the large number of partonic subprocesses included in the calculation. These are from the quark and gluon content of the initial hadrons, and from CKM quark mixing. For example, there are 180 subprocesses which contribute to the $W+2$ jets process, and 292 subprocesses in $W+3$ jets production at the LHC, even when quarks from only the first two generations are taken into account.

We propose a simple modification of the rules for evaluation of cross sections and distributions, which avoids multiplication of channels from the mixture of quark states. The method is based on a unitary rotation of down quarks, thus, transporting the mixing matrix elements from vertices of Feynman diagrams to the parton distribution functions (PDF). As a result, one can calculate cross sections with significantly fewer subprocesses. For the example mentioned above, with the new rules, one need evaluate only 21 and 33 subprocesses respectively. The matrix elements of the subprocesses are calculated without quark mixing but with a modified PDF convolution which depends on the quark mixing angle, and on the topologies of gauge invariant classes of diagrams. The proposed method has been incorporated into the CompHEP program and checked with various examples.
\end{abstract}




\section{Introduction}

The evaluation of cross sections for hadron collisions typically involves a large number of subprocesses. The reason for this is the quark and gluon content of the initial hadrons and the Cabbibo-Kobayashi-Maskava (CKM) mixing of the down quarks. At Tevatron and LHC energies in many cases 5 flavors, $u, d, c, s$ and $b$, give a sizable contribution through the corresponding parton densities. Note that subprocesses sometimes contribute only because of the non-diagonality of the CKM matrix. An example is the subprocess $u \bar{d} \rightarrow s \bar{d} W^{+}$which contributes to the $W+2$ jets production. Furthermore, the number of subprocesses increases with the number of quarks in the final state, in particular, because the final state jets produced by various subprocesses are indistinguishable $\mathrm{t}$. The process of $W$-boson and jet production (an important background to various Standard Model and "new physics" processes) exemplifies the problem: 180 subprocesses contribute to $W+2 j e t s$ and 292 subprocesses to $W+3$ jets production if only the quarks of first two generations and only QCD diagrams are taken into account.

The huge multiplication of channels stands as a real computation problem despite the fact that the matrix elements of some subprocesses may have similar analytical structures. Indeed, one should separately organize Monte Carlo integration and/or event generation for each subprocess separately because the convolution with the parton distribution functions (PDF) is flavour dependent. This is a present-day problem for automatic calculations of collision processes (see e.g. [1] and references therein for review of this new computation approach).

In this letter we propose a new method which simplifies the flavour combinatorics and reduces the multiplication of channels due to the mixture of quark states. Implementation of the proposed algorithm in Monte Carlo codes is straightforward. It has been incorporated into the CompHEP program [4] and checked for many examples. In the method the quarks of the first two generations are taken to be massless and do not mix with the third generation which is obviously a good approximation for many processes in the Tevatron and LHC energy range. The method is based on a rotation of down quarks, thus, transporting the mixing matrix elements from vertices of subprocess Feynman diagrams to the parton distribution functions. The complete set of subprocess diagrams are divided into gauge invariant classes, and depending on the topology of the class, new computation rules are formulated. The method drastically simplifies the calculation of matrix elements but leads to a modification of the PDF convolution procedure. We demonstrate the power of the proposed technique with the example of $W+2 j e t s$ production at LHC. Here only 21 subprocesses have to be evaluated with the new technique, which should be compared with 180 subprocesses in the standard approach.

The paper is organized as follows. In Section 1 we discuss CKM diagonalization at the level of Feynman amplitudes and introduce basic notations. Here the topologies of the gauge invariant classes of diagrams are defined, for which in Section 2 new PDF convolution rules are formulated. The $W+2 j e t s$ production process at LHC is discussed in Section 3. In the Appendix we briefly comment on the implementation of the new rules in the CompHEP program.

\footnotetext{
${ }^{1}$ An important exclusion is a $b$-quark jet which can be distinguished from light quark jets with a micro-vertex detector.
} 


\section{Feynman amplitudes}

Let us consider a parton subprocess with a quark in the initial state. There are two possible topologies of any Feynman diagram with the corresponding quark line: 1) the quark line goes through the diagram from the initial to final state - "scattering topology", and 2) the quark line connects both initial state partons - "annihilation topology". In Fig.11 and Fig.2a one can see examples of scattering topology, and diagrams of annihilation topology are shown in Fig.2b,c. Here the interior of the circle represents the diagram, and the solid line represents a quark line (quark current). An important point is that according to the theorem proved in [2], the two sets of diagrams with the scattering and annihilation topologies form gauge invariant classes with respect to the SM gauge group. Thus, one can change the computational rules for each class independently. Note, that a specific parton subprocess could contain only scattering topology diagrams, or only annihilation topology diagrams, or both. For the process $u \bar{d} \rightarrow d \bar{d} W^{+}$shown in Fig. 3 both annihilation and scattering diagrams contribute. For our further consideration it is necessary to determine whether the quark current is charged (CC) or neutral (NC). This is done by counting the quark vertices with $W$-bosons. The charged current involves an odd and the neutral current an even number of $W$ vertices. Both cases might include an arbitrary number of vertices with gluons, photons and Z-bosons.

Two approximations are used in our method. We neglect: 1)the masses of quarks from the two first generations, and 2) the mixing with the third generation. Thus, the $3 \times 3$ CKM mixing matrix reduces to the $2 \times 2$ Cabbibo matrix:

$$
V_{C K M} \Longrightarrow\left(\begin{array}{cc}
V & 0 \\
0 & 1
\end{array}\right), \quad V=\left(\begin{array}{cc}
\cos \vartheta_{c} & \sin \vartheta_{c} \\
-\sin \vartheta_{c} & \cos \vartheta_{c}
\end{array}\right)
$$

where $\vartheta_{c}$ is the Cabbibo angle.

The zero quark mass limit is a good approximation in many applications, in particular, when one evaluates matrix elements of hard subprocesses. Indeed, the energy scale, e.g. the partonic collision energy, for Tevatron-LHC hard subprocesses is $\mathcal{O}(100) \mathrm{GeV}$ and higher. Then, minimal energy-like cuts on jets (momentum transfer, invariant masses of jet pairs etc.) are of order $\mathcal{O}(10) \mathrm{GeV}$ or more. This means that the momenta in the propagators are of the same order and mass effects can be neglected in matrix elements. At the same time, the flavour dependence of parton distributions is sizable and can substantially affect observables.

The second approximation (no mixing with the third generation) also works well in many applications. The $V_{t b}$ mixing matrix element is very close to unity and the nondiagonal CKM matrix elements for bottom and top quarks are very small. Of course, these elements are responsible for important physical phenomena, such as B-meson oscillations and rare decays of heavy mesons. In these cases the proposed method can, obviously, not be applied. Another reason why the second approximation is reasonable is that $t$ and $b$ quarks produce final state objects in a detector which are very different from light quark jets: the $b$ quark jet has a secondary vertex, and the $t$-quark appears as a heavy narrow resonance.

The starting point of our consideration is the diagonalization of the quark mixing matrix in vertices. This means that rotated down quark states are used in Feynman 
rules, $d^{\prime}=d \cos \vartheta_{c}+s \sin \vartheta_{c}$ and $s^{\prime}=-d \sin \vartheta_{c}+s \cos \vartheta_{c}$ rather than $d$ and $s$ states being eigenstates of the mass matrix. It is worth recalling that all electroweak vertices are diagonal over the isodoublets $\left(\begin{array}{c}u \\ d^{\prime}\end{array}\right)_{L}$ and $\left(\begin{array}{c}c \\ s^{\prime}\end{array}\right)_{L}$. For example, the charged current (CC) electroweak vertex is diagonal in this rotated basis,

$$
W_{\mu}^{+} \cdot J_{C C}^{\mu}=W_{\mu}^{+} \cdot \bar{u}_{i}^{L} \gamma^{\mu} V_{i j} d_{j}{ }^{L}=W_{\mu}^{+} \cdot \bar{u}_{i}^{L} \gamma^{\mu} d_{j}^{L}
$$

Here $d_{i}^{\prime}=V_{i j} d_{j}$ (where $i, j=1,2$ are the generation indices, so $d=d_{1}$ and $s=d_{2}$ ). As a result, elements of the CKM mixing matrix do not enter in the matrix element if one calculates in terms of these rotated down quarks.

Let us consider an electroweak model with the only one generation of quarks (referred to as the $E W_{u d}$ model), and denote generalized up and down quarks by $q_{u}$ and $q_{d}$ respectively. Then, Feynman amplitudes in the EW model with two generations and Cabbibo mixing can be evaluated in the $E W_{u d}$ model with a single quark generation. Indeed, in the $E W_{u d}$ model, the CC vertex represents the Lagrangian term $W_{\mu}^{+}{\overline{q_{u}}}^{L} \gamma^{\mu} q_{d}{ }^{L}$, and its contribution to the amplitude is analytically the same as the contribution of the standard $\mathrm{CC}$ vertex (remember that we have neglected quark masses). The only difference is a multiplication of some amplitudes by mixing matrix elements. There are only two generic variants for the case of scattering topology diagrams illustrated in Fig.1: CC in Fig. 17a and NC in Fig.1 1b. One can easily see that the rotation of down quarks results in only one factor $V_{i j}$ (or $V_{i j}^{-1}$ in conjugated cases) in front of the each CC line, while the mixing matrix elements cancel each other inside the $\mathrm{NC}$ line due to the unitarity of the mixing matrix $\sum_{k} V_{i k}^{-1} V_{k j}=\delta_{i j}$. One should stress that the summation over internal flavours in propagators is performed when the amplitude is evaluated. In the Fig.1 only one $W$ vertex is shown in the $\mathrm{CC}$ case and two $W$ vertices in the $\mathrm{NC}$ case. However the statements are correct for the general $\mathrm{CC}$ and $\mathrm{NC}$ cases. Obviously the same conclusions are valid in the case of CC and NC diagrams of the annihilation topology as shown in Fig.2 $\mathrm{b}, \mathrm{c}$.

An important difference between the annihilation and scattering topologies appears at the level of squared diagrams when the convolution with the parton distribution functions is performed.

\section{Squared diagrams and PDF convolution}

The next step is to square the matrix element and convolute with the PDF's. Here we derive four basic rules for the technique under discussion.

\section{Scattering topology. 1st Rule}

Let us consider Feynman diagrams with the scattering topology. The second in-state can be a quark, an anti-quark or a gluon. We denote the sum of this type of Feynman diagrams as $\mathcal{D}_{s c}$. An example is presented in Fig. 2 2 a for the case of a CC upper quark line and an NC lower quark line. After squaring of diagrams of this type and convoluting with PDF's one can write the contribution to the cross section in the form (for the example in the Fig. 2a):

$$
\left|\mathcal{D}_{s c}\right|^{2} \Longrightarrow \sum_{i j k n} \int d x_{1} d x_{2} f_{d_{i}}\left(x_{1}\right) f_{u_{j}}\left(x_{2}\right)\left|M_{i j k n}\right|^{2}=
$$




$$
=\int d x_{1} d x_{2}\left[\sum_{i k} f_{d_{i}}\left(x_{1}\right) V_{i k} V_{k i}^{-1}\right]\left[\sum_{j n} f_{u_{j}}\left(x_{2}\right) \delta_{j n} \delta_{n j}\right]|\mathcal{M}|^{2},
$$

where by $M$ and $\mathcal{M}$ we denote matrix elements evaluated in the standard EW theory and in the $E W_{u d}$ model respectively. Due to the unitarity of the mixing matrix one has $V_{i k} V_{k i}^{-1}=\delta_{i i}$, and the first rule can be written as

$$
\left|\mathcal{D}_{s c}\right|^{2} \Longrightarrow \int d x_{1} d x_{2}\left[f_{d}\left(x_{1}\right)+f_{s}\left(x_{1}\right)\right]\left[f_{\bar{u}}\left(x_{2}\right)+f_{\bar{c}}\left(x_{2}\right)\right]|\mathcal{M}|^{2}
$$

This means that one can evaluate squared diagrams of the scattering topology class with only one quark generation, but should convolute the corresponding gauge invariant squared matrix element with modified structure function(s) - a sum of down (or up) PDF's.

\section{Annihilation CC topology. 2nd Rule}

In the case of the annihilation topology the $\mathrm{CC}$ and $\mathrm{NC}$ cases lead to different rules for convolution with the PDF. We start from the CC annihilation case which is generically shown in Fig. 2 $\mathrm{b}$. Recall that this variant occurs only if the quark line has an odd number of $W$ vertices. We denote the sum of this class of Feynman diagrams as $\mathcal{D}_{a}^{C C}$. When one squares this sum of diagrams and convolutes with the PDF, one gets:

$$
\begin{aligned}
\left|\mathcal{D}_{a}^{C C}\right|^{2} & \Longrightarrow \sum_{i j} \int d x_{1} d x_{2} f_{d_{i}}\left(x_{1}\right) f_{\bar{u}_{j}}\left(x_{2}\right)\left|M_{i j}\right|^{2} \\
& =\int d x_{1} d x_{2}\left[\sum_{i j} f_{d_{i}}\left(x_{1}\right) f_{\bar{u}_{j}}\left(x_{2}\right) V_{i j} V_{j i}^{-1}\right]|\mathcal{M}|^{2} .
\end{aligned}
$$

In contrast to the scattering case here one can not use the unitarity condition to cancel two elements of the mixing matrix because the summations over the indices, $i$ and $j$, also include the structure functions $f_{d_{i}}$ and $f_{\bar{u}_{j}}$. Thus, the second rule can be written in the following form (see the example in Fig. 20 b):

$$
\begin{aligned}
\left|\mathcal{D}_{a}^{C C}\right|^{2} \Longrightarrow \int d x_{1} d x_{2} & {\left[f_{d}\left(x_{1}\right) f_{\bar{u}}\left(x_{2}\right) \cos ^{2} \vartheta_{c}+f_{s}\left(x_{1}\right) f_{\bar{c}}\left(x_{2}\right) \cos ^{2} \vartheta_{c}+\right.} \\
+ & \left.f_{d}\left(x_{1}\right) f_{\bar{c}}\left(x_{2}\right) \sin ^{2} \vartheta_{c}+f_{s}\left(x_{1}\right) f_{\bar{u}}\left(x_{2}\right) \sin ^{2} \vartheta_{c}\right]|\mathcal{M}|^{2} .
\end{aligned}
$$

where we have explicitly expressed the mixing matrix elements in terms of the Cabbibo mixing angle.

One can see that the annihilation-type contribution to the squared matrix element is convoluted with non-factorizable products of PDF's.

\section{Annihilation NC topology. 3d Rule}

We denote the sum of all diagrams with the annihilation $\mathrm{NC}$ quark line as $\mathcal{D}_{a}^{N C}$. The generic example is shown in Fig. 2cc. In this case the quark line has an even number of $W$-boson vertices. In the same way as above one can derive the following formula:

$$
\left|\mathcal{D}_{a}^{N C}\right|^{2} \Longrightarrow \sum_{i j} \int d x_{1} d x_{2} f_{d_{i}}\left(x_{1}\right) f_{\bar{d}_{j}}\left(x_{2}\right)\left|M_{i j}\right|^{2}=
$$




$$
=\int d x_{1} d x_{2}\left[\sum_{i j} f_{d_{i}}\left(x_{1}\right) f_{\bar{d}_{j}}\left(x_{2}\right) \delta_{i j} \delta_{j i}\right]|\mathcal{M}|^{2} .
$$

Therefore, the third rule can be written in the form (if the in-quarks are down):

$$
\left|\mathcal{D}_{a}^{N C}\right|^{2} \Longrightarrow \int d x_{1} d x_{2}\left[f_{d}\left(x_{1}\right) f_{\bar{d}}\left(x_{2}\right)+f_{s}\left(x_{1}\right) f_{\bar{s}}\left(x_{2}\right)\right]|\mathcal{M}|^{2}
$$

with the obvious generalization for up quarks.

\section{Interference of $\mathcal{D}_{s c}$ and $\mathcal{D}_{a}$ topologies}

In the general case diagrams of both, scattering and annihilation, topologies could contribute. The interference between gauge invariant diagram classes with these topologies is also gauge invariant and, therefore, can be independently convoluted with the PDF. As in the above derivations the PDF convolution for the interference of $\mathcal{D}_{s c}$ diagrams with $\mathcal{D}_{a}^{C C}$ diagrams is given by the same formula as for $\left|\mathcal{D}_{a}^{C C}\right|^{2}$ (2nd Rule), and for the interference of $\mathcal{D}_{s c}$ with $\mathcal{D}_{a}^{N C}$ by the corresponding formula for $\left|\mathcal{D}_{a}^{N C}\right|^{2}$ (3rd Rule).

\section{The final state quark-antiquark line. 4th Rule}

Finally let us consider Feynman diagrams where a quark line connects two out-states, as in Fig. 2d. Here the summation over generation indices does not involve parton distribution functions and can, therefore, be performed explicitly. The result is that the contribution of the corresponding squared diagrams, evaluated in the $E W_{u d}$ model, should be multiplied by two for both the $\mathrm{CC}$ and $\mathrm{NC}$ cases. Indeed, according to Fig. 2 $\mathrm{d}$ each of these summations give in the squared diagram the factors: $\sum_{i j} \delta_{i j} \delta_{j i}=2$ in the $\mathrm{NC}$ and $\sum_{i j} V_{i j} V_{j i}^{-1}=\sum_{i j} \delta_{i j}=2$ in the CC cases.

Note that the 4th Rule is valid not only in cases where the quark loop in squared diagrams connects out-state(s) and never passes through in-state(s), but also for each quark loop in next-to-leading corrections.

Of course, the 4th Rule is valid only under the assumption that the fragmentation of the four light quarks and antiquarks leads to indistinguishable jets. If one includes nontrivial fragmentation functions, e.g. for a $c$-quark, all the above rules have to be modified. We do not present here the corresponding formulas which however could be easily derived.

\section{Test: $W+2$ jets production at $\mathbf{L H C}$}

In this section we illustrate the proposed technique with the example of $\mathrm{W}^{+}+2$ jets production at LHC. Here a total of 180 subprocesses contribute in the standard technique when all four light quarks contribute separately and the CKM matrix is present in $W$ boson vertices. With the new technique only 21 subprocesses need to be evaluated.

We shall not discuss this example in full detail but present the results of a numerical test for the $u \bar{d} \rightarrow d \bar{d} W^{+}$subprocess with permutations of quarks within pairs $(u, c)$ and $(d, s)$. Note that in this example three rules are used: 1st, 2nd and 4th. In the standard technique 12 subprocesses are involved and the corresponding contributions to the cross section are collected in Tab. 1 for two values of the kinematical cut on the transverse momenta of the final partons and the $W$ boson, $p_{T}^{j e t}>p_{T}^{0}$ and $p_{T}^{W}>p_{T}^{0}$ with $p_{T}^{0}=20$ 
or $200 \mathrm{GeV}$. The cross sections were calculated in the Standard Model with the averaged values for CKM matrix elements and quark masses taken from the Particle Data Group [3]. For calculations the CompHEP program [4] has been used, and the accumulated MC error in all cases was less than $0.6 \%$. We neglect the contributions of subleading diagrams with electroweak boson propagators, calculating the cross section to leading $\alpha \alpha_{s}^{2}$ order.

Using the new method one should evaluates only one subprocess in the one quark doublet $E W_{u d}$ model, $q_{u} \overline{q_{d}} \rightarrow q_{d} \overline{q_{d}} W^{+}$. The corresponding Feynman diagrams are shown in Fig. 3. This subprocess is of the mixed type where two gauge invariant classes of diagrams: the annihilation CC topology (Fig. Ba) and the scattering topology (Fig. 3b), contribute. The 1st Rule is used for the squared scattering topology contribution. The $2 \mathrm{nd}$ Rule is used for the squared annihilation CC topology contribution with a multiplication by the factor 2 according to the 4th Rule. Finally, the 2 nd Rule is used to evaluate the interference between the two gauge invariant classes of Feynman diagrams. We have calculated all three contributions to the cross section of the subprocess $q_{u} \overline{q_{d}} \rightarrow q_{d} \overline{q_{d}} W^{+}$ using CompHEP code in which the $E W_{u d}$ model and Rules 1-3 have been implemented (see the Appendix for details). The results (see Tab. 2) for the total rate, $\sigma\left(p_{T}>20 \mathrm{GeV}\right)=$ $112.95 \mathrm{pb}$ and $\sigma\left(p_{T}>200 \mathrm{GeV}\right)=0.30213 \mathrm{pb}$, are in an agreement with the "standard" calculations of Tab. 1 within the statistical error of less than $0.6 \%$.

\section{Conclusions}

We have shown that hard collision processes at hadron colliders can be evaluated in an economical way, greatly reducing the number of contributing subprocesses. The proposed computational technique can be applied only if the quark masses of the first two generations and mixing with the 3rd generation can be neglected. These assumptions are valid for most applications at the Tevatron and LHC. In the proposed technique the Standard Model with a single generation of up and down quarks is used and a squared matrix element is evaluated without involving elements of the mixing matrix. The resulting matrix squared matrix element is convoluted with modified parton distribution functions according to formulas given above as Rules 1-4. Each of these Rules corresponds to a gauge invariant class of squared diagrams.

In Rule 1 the squared matrix element is convoluted with $f_{u}(x)+f_{c}(x)$ or $f_{d}(x)+f_{s}(x)$ for an in-state of up-type or down-type quarks respectively However, in the cases of Rules 2 and 3 the squared matrix element is convoluted over Bjorken variables $x_{1}$ and $x_{2}$ with a non-factorizable function. When applying the 2nd Rule the function

$$
\left[f_{d}\left(x_{1}\right) f_{\bar{u}}\left(x_{2}\right)+f_{s}\left(x_{1}\right) f_{\bar{c}}\left(x_{2}\right)\right] \cos ^{2} \vartheta_{c}+\left[f_{d}\left(x_{1}\right) f_{\bar{c}}\left(x_{2}\right)+f_{s}\left(x_{1}\right) f_{\bar{u}}\left(x_{2}\right)\right] \sin ^{2} \vartheta_{c}
$$

or a similar function where the substitution $d \rightarrow \bar{d}$ and $\bar{u} \rightarrow u$ are made, is used. In the case of the 3rd Rule the convolution is with either

$$
\begin{gathered}
f_{u}\left(x_{1}\right) f_{\bar{u}}\left(x_{2}\right)+f_{c}\left(x_{1}\right) f_{\bar{c}}\left(x_{2}\right) \quad \text { or }, \\
f_{d}\left(x_{1}\right) f_{\bar{d}}\left(x_{2}\right)+f_{s}\left(x_{1}\right) f_{\bar{s}}\left(x_{2}\right),
\end{gathered}
$$

depending on which quark-antiquark pair occurs in the in-state. 
The authors are indebted to A.E. Pukhov, Th. Ohl and B. Straub for useful discussions. This work was partially supported by the CERN-INTAS 99-377, RFBR-DFG 99-02-04011 and RFBR 00-01-00704 grants, by the Russian Ministry of Science and Technology, by St.Petersburg Grant Center and by the program "Universities of Russia" (grant 990588).

\section{References}

[1] Proceedings of "Physics at TEV colliders" Workshop (Les Houches, June 1999), QCD working group, to appear.

Proceedings of the "Standard Model Physics (and more) at the LHC" Workshop (CERN, 1999), QCD working group report, to appear.

[2] E. Boos and T.Ohl, Phys. Rev. Lett. 83 (1999) 480.

[3] Review of Particle Properties, Phys. Rev. D54 (1996) .

[4] A.Pukhov et al., CompHEP user's manual, v.3.3. INP MSU 98-41/542; hep-ph/9908288.

[5] http://home.cern.ch/v/vilyin/public/html/v33.23.tar.gz

\section{Appendix}

The Rules derived in this letter have been implemented in the CompHEP code v.33. New model, referred to above as $E W_{u d}$, was created on the base of the SM where only one quark generation, up and down quarks denoted as $q_{u}$ and $q_{d}$ was kept without any CKM matrix elements. The masses of these generalized quarks were set to zero. Then, the option for numerical convolution of squared diagrams with parton distribution functions was modified in accordance with Rules 1-3. The code of this version of CompHEP is available from the Web [5].

In the most general case the user has to subdivide the whole set of squared diagrams into two parts: 1) $\left|\mathcal{D}_{s c}\right|^{2}$, and 2) $\left|\mathcal{D}_{a}\right|^{2}$ plus the interference diagrams $2 \operatorname{Re}\left(\mathcal{D}_{s c} \cdot \mathcal{D}_{a}^{*}\right)$. Each of these parts should be calculated separately. In particular, for each part the user has to set the variable "PDFfactor" in the menu option "User menu" as follows: PDF factor $=1$ for $\left|\mathcal{D}_{s c}\right|^{2}$ (referred to in CompHEP as the "t-channel" case), and PDF factor $=0$ for $\left|\mathcal{D}_{a}\right|^{2}+2 \operatorname{Re}\left(\mathcal{D}_{s c} \cdot \mathcal{D}_{a}^{*}\right)$ (referred to in CompHEP as the "s-channel" case). The program automatically recognizes which Rule, 2nd or 3rd, should be used in the latter case.

Rule 4 (multiplication by a factor 2) has to be applied by hand if the corresponding quark line is presented in a squared diagram.

One should note that this is not a completely automatic realization of Rules 1-4. This is planned for future CompHEP development. 
Figures

a)
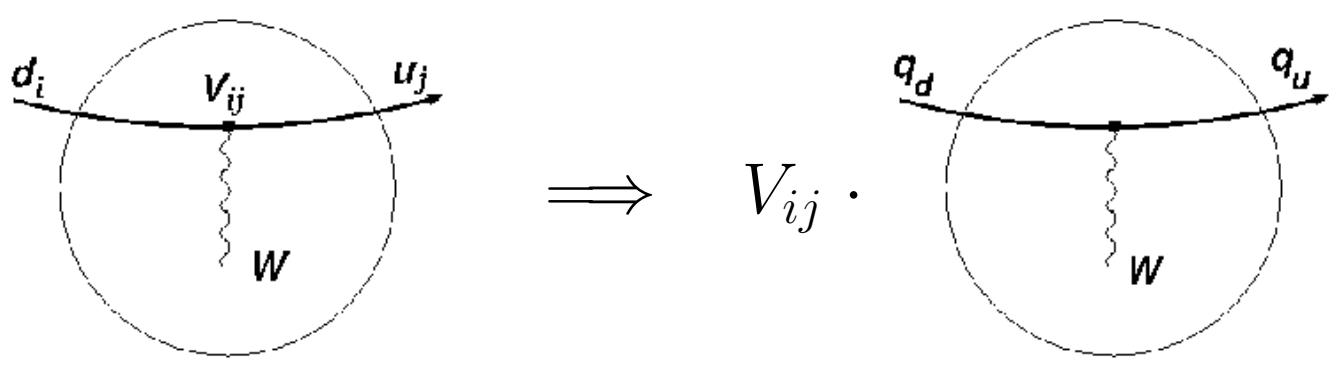

b)
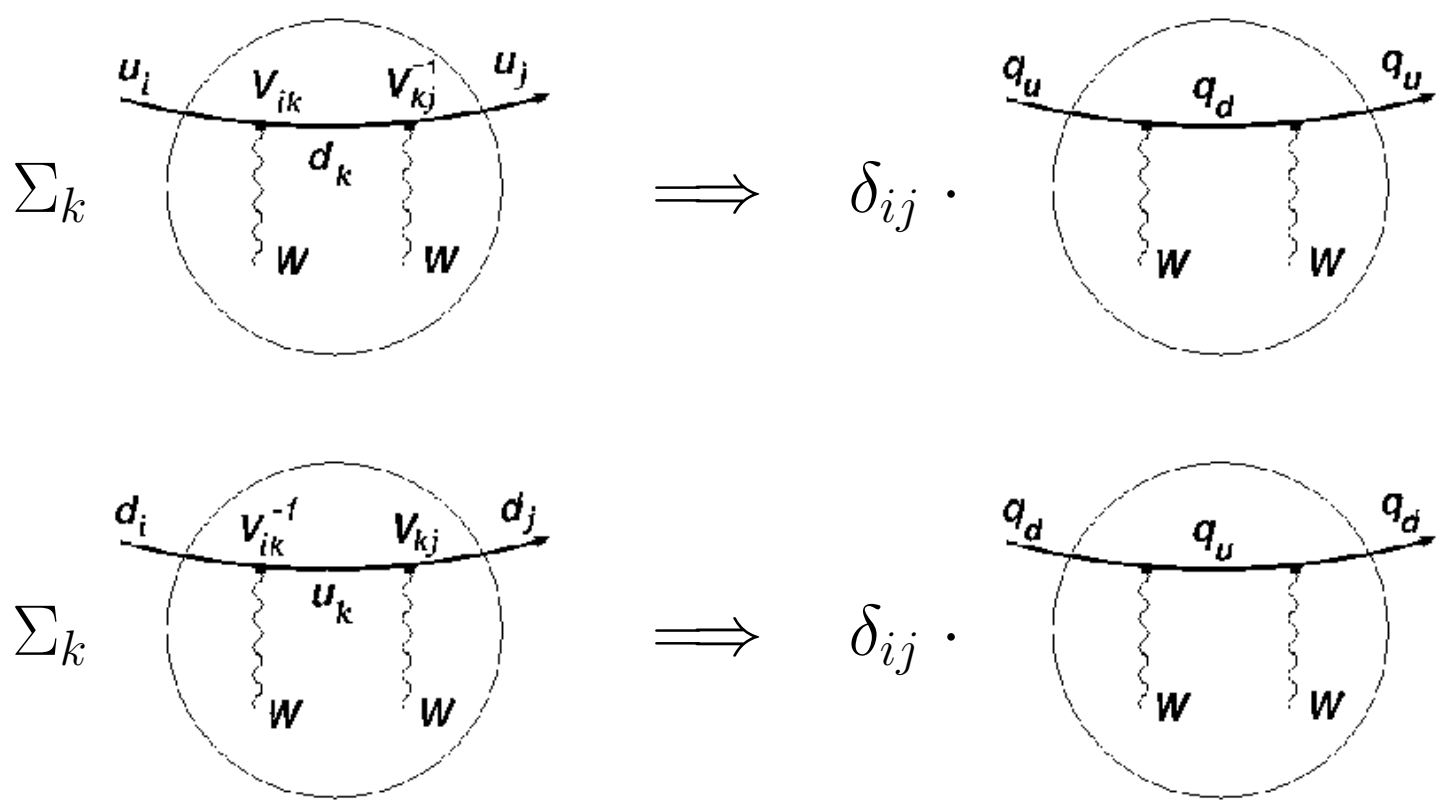

Figure 1: Generic scattering topology diagrams with: a) a single $W$ vertex and b) two $W$ vertices on the quark line. No summation over the generation indices $i$ and $j$ is implied. Here and in the next figure the external lines on the left and right sides of the diagram correspond to in- and out-states respectively. 
a)

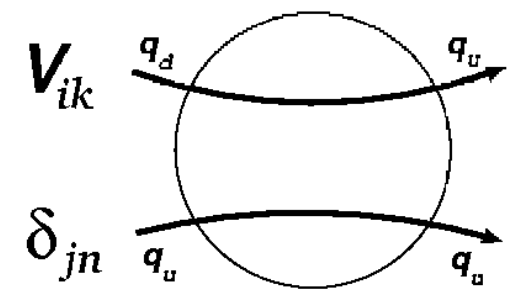

c)

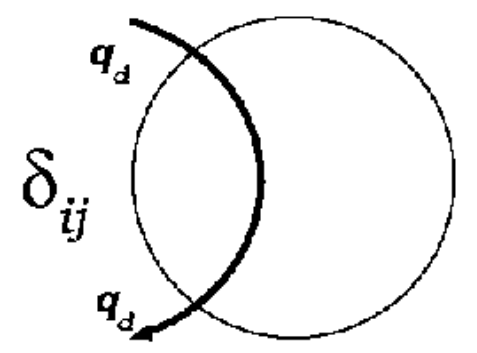

b)

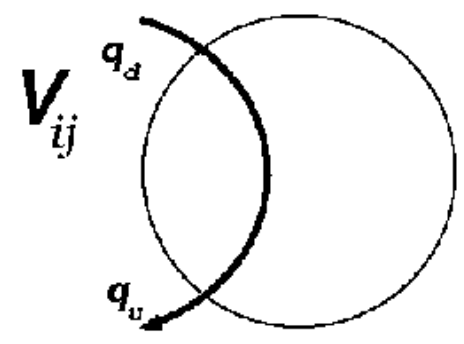

d)

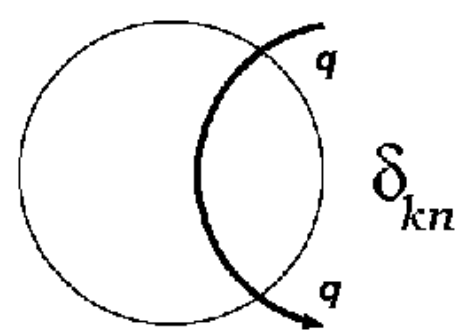

Figure 2: Generic diagrams with different topologies in which the quark lines connect inand out-states. Here $i$ and $j$ are the generation indices of the $i n$-quarks, while $k$ and $n$ are the generation indices for out-quarks. Diagram d) includes a quark line connecting two out-states.

a)
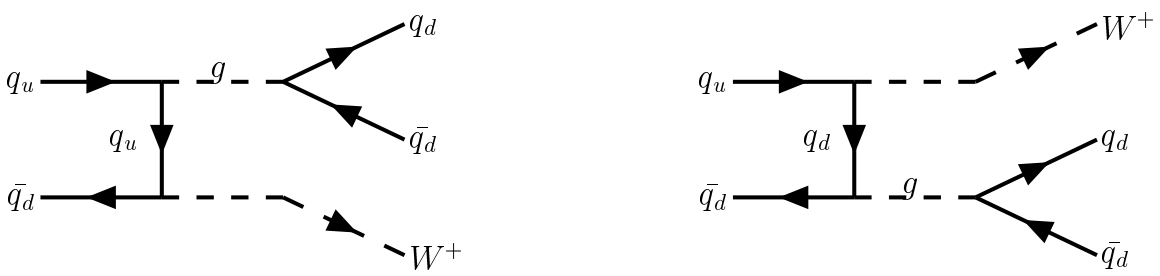

b)
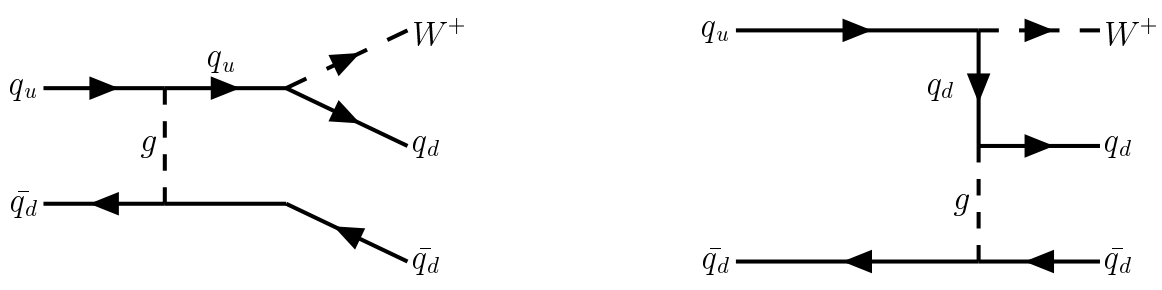

Figure 3: Feynman diagrams for the subprocess $q_{u} \overline{q_{d}} \rightarrow q_{d} \overline{q_{d}} W^{+}$which contribute to $W^{+}+2$ jets production at LHC. Case a) corresponds to the $\mathcal{D}_{a}^{C C}$ topology of diagrams, while case b) corresponds to the $\mathcal{D}_{s c}$ topology. 


\section{Tables}

\begin{tabular}{|c|ll|}
\hline \multirow{2}{*}{ Subprocess } & \multicolumn{2}{|c|}{ cross section, pb } \\
\cline { 2 - 3 } & $p_{T}^{0}=20 \mathrm{GeV}$ & $p_{T}^{0}=200 \mathrm{GeV}$ \\
\hline$u d \rightarrow s \bar{s} W^{+}$ & 9.368 & 0.01746 \\
$u \bar{d} \rightarrow \bar{d} s W^{+}$ & 2.315 & 0.007807 \\
$u \bar{d} \rightarrow d \bar{d} W^{+}$ & 55.84 & 0.1733 \\
$u \bar{s} \rightarrow s \bar{s} W^{+}$ & 2.113 & 0.005301 \\
$u \bar{s} \rightarrow d \bar{s} W^{+}$ & 32.67 & 0.09074 \\
$u \bar{s} \rightarrow d \bar{d} W^{+}$ & 0.3748 & 0.0005516 \\
$c \bar{d} \rightarrow s \bar{s} W^{+}$ & 0.0739 & 0.00003557 \\
$c \bar{d} \rightarrow \bar{d} s W^{+}$ & 4.601 & 0.004845 \\
$c \bar{d} \rightarrow d \bar{d} W^{+}$ & 0.3206 & 0.0002915 \\
$c \bar{s} \rightarrow s \bar{s} W^{+}$ & 4.093 & 0.003090 \\
$c \bar{s} \rightarrow d \bar{s} W^{+}$ & 0.1528 & 0.0001359 \\
$c \bar{s} \rightarrow d \bar{d} W^{+}$ & 0.9842 & 0.0003853 \\
\hline In total & 112.90 & 0.30391 \\
\hline
\end{tabular}

Table 1: Contributions, in pb, of different $q \bar{q}$ channels to the $W^{+}+2 j e t s$ production cross section at LHC. In addition to cuts on transverse momenta, $p_{T}^{j e t}>p_{T}^{0}$ and $p_{T}^{W}>p_{T}^{0}$, the following cuts were applied: on pseudorapidity $\left|\eta_{\text {jet }}\right|<5$ and on the jet separation in the $\eta-\varphi$ plane $R\left(j_{e t}, j e t^{\prime}\right)>0.5$. The statistical accuracy of the MC calculations is better than $0.6 \%$.

\begin{tabular}{|c|ll|}
\hline Classes of squared diagrams, & \multicolumn{2}{|l|}{ contribution to cross section, pb } \\
\cline { 2 - 3 } used Rules & $p_{T}^{0}=20 \mathrm{GeV}$ & $p_{T}^{0}=200 \mathrm{GeV}$ \\
\hline$\left|\mathcal{D}_{s c}\right|^{2}, 1$ st Rule & 89.76 & 0.2621 \\
$\left|c a l D_{a}^{C C}\right|^{2}, 2$ nd +4 th Rules & 21.62 & 0.03692 \\
$2 R e\left(\mathcal{D}_{a}^{C C} \cdot \mathcal{D}_{s c}^{*}\right)$, 2nd Rule & 1.549 & 0.003111 \\
\hline In total & 112.93 & 0.30213 \\
\hline
\end{tabular}

Table 2: Contribution, in pb, of the subprocess $q_{u} \overline{q_{d}} \rightarrow q_{d} \overline{q_{d}} W^{+}$to the $W^{+}+2$ jets production cross section at LHC, evaluated with the help of Rules 1,2 and 4 using the of $E W_{u d}$ model. The same cuts were applied as in Tab. 1. The accuracy of the MC calculations is better than $0.6 \%$. 